\title{
Diabetic muscle infarction with complications
}

\author{
Sarah Olivier Cabrera, Raquel Michel, Aracelis Lu, Vihren Dimitrov
}

\begin{abstract}
Introduction: Diabetic muscle infarction (DMI) is a rare complication of diabetes mellitus (DM) that is often misdiagnosed. We report a case of diabetic muscle infarction and discuss diagnostic testing and treatment modalities to avoid complications. Case Report: A 50-year-old male presented with spontaneous left thigh swelling for five days. He had several previous episodes in the contralateral side with recurrent admissions. His past medical history consisted of poorly controlled type 2 DM on insulin therapy, end stage renal diseases on hemodialysis and hypothyroidism. Physical examination revealed a tight, warm, erythematous and tender left thigh. Laboratory examination showed white blood cell count $1.23 \times 10^{4} / \mu \mathrm{L}$ with neutrophilia; hemoglobin 10.7 g/L, hematocrit 32.3\%, alkaline phosphatase 792 $\mathrm{U} / \mathrm{L}$, creatinine $5.94 \mathrm{mg} / \mathrm{dL}$ and glucose 455 $\mathrm{mg} / \mathrm{dL}$. Venous ultrasound of left lower extremity revealed deep vein thrombosis in the mid left femoral vein however, venogram was negative. Compartment syndrome was considered and fasciotomy with biopsy was performed. Subsequently the patient worsened which lead to multiple re-interventions. After reviewing medical records from both our
\end{abstract}

Sarah Olivier Cabrera ${ }^{1}$, Raquel Michel ${ }^{1}$, Aracelis Lu², Vihren Dimitrov ${ }^{1}$

Affiliations: 'Department of Internal Medicine, Lincoln Medical and Mental Health Center, Bronx, New York, USA; ${ }^{2}$ Department of Psychiatry, Lincoln Medical and Mental Health Center, Bronx, New York, USA.

Corresponding Author: Vihren Dimitrov, MD, 234 East 149th Street, Bronx, NY, USA. 10451; Ph: 718-579-5016/5278; Fax: 718-579-4836; Email: Vihren.Dimitrov@nychhc.org

Received: 14 September 2011

Accepted: 12 August 2012

Published: 01 June 2013 hospital and from other facilities, a diagnosis of DMI was established. Muscle biopsy supported this, revealing active macrophage infiltration with areas of necrosis. Treatment is conservative with pain management, rest and proper glycemic control. Surgical intervention is not necessary as it increases morbidity. Conclusion: Although rare, DMI is a serious and potentially disabling complication of uncontrolled DM which continues to be underdiagnosed. Recognizing DMI more efficiently could prevent the patient from undergoing numerous, unnecessary diagnostic procedures, and medical or surgical interventions which in turn can result in more harm than good for the patient.

Keywords: Diabetic myonecrosis, Muscular degeneration, Diabetic microangiopathy

$$
* * * * * * * * *
$$

Cabrera SO, Michel R, Lu A, Dimitrov V. Diabetic muscle infarction with complications. International Journal of Case Reports and Images 2013;4(6):303-307.

doi:10.5348/ijcri-2013-06-318-CR-3

\section{INTRODUCTION}

Diabetic muscle infarction (DMI) is a rare complication of diabetes mellitus (DM) that is often misdiagnosed. DMI usually affects the muscles of the thighs and calves with rare reported cases in the upper extremities. It presents as sudden onset leg pain and swelling and becomes a diagnostic challenge. T2weighed magnetic resonance imaging (MRI) is the diagnostic imaging of choice and while muscle biopsy provides a definitive diagnosis, surgical intervention is 
neither necessary nor recommended given the fact that it leads to more complications in these patients. Such complications include hemorrhages within the affected muscle, often requiring blood transfusions [1]. DMI resolves spontaneously and no particular treatment modality has proven effective. Pain management and complete rest is the mainstay of therapy.

\section{CASE REPORT}

A 50-year-old male presented to the emergency department complaining of left thigh pain, swelling and redness for last five days. There was no history of trauma but similar episodes of leg pain had occurred since 2007 (twice in the right thigh and once in the left thigh), resulting in multiple admissions. Vascular ultrasound did not produce any definitive diagnosis and it was assessed as cellulites.

Past medical history included uncontrolled type 2 DM, end stage renal diseases (ESRD) on hemodialysis, anemia of chronic disease, hypothyroidism and depression. He had a 90 pack-year smoking history (quit seven months prior to last admission) and history of alcohol abuse. His medications included lisinopril, metoprolol, levothyroxyne, citalopram, and both fast and long acting insulin.

Physical examination revealed moderately tender left anterior thigh, fullness and tightness of left lateral compartments with erythema and increased warmth in comparison to the right thigh. The laboratory examination revealed: hemoglobin $10.7 \mathrm{~g} / \mathrm{dL}$, hematocrit $32.3 \%$, white blood cell count $1.23 \times 10^{4} / \mu \mathrm{L}$ with a left shift in WBC's, prothrombin time 12 seconds, international normalized ratio 1.2, alkaline phosphatase $792 \mathrm{U} / \mathrm{L}$, creatinine $5.94 \mathrm{mg} / \mathrm{dL}$, and glucose 455 $\mathrm{mg} / \mathrm{dL}$.

A vascular ultrasound of the left lower extremity (LLE) during admission demonstrated noncompressible echogenic focus most consistent with deep venous thromboembolism (DVT) in the mid left femoral vein (Figure 1). The patient was admitted with a diagnosis of DVT, started on anticoagulation protocol and was evaluated by interventional radiology (IR) service for thrombolysis/thrombectomy with Trellis Device $^{\circledR}$. The LLE venogram was negative, therefore no intervention was needed (Figure 2). Anticoagulation was continued and the patient continued to complain of LLE pain with increased swelling. Surgery was consulted and a diagnosis of compartment syndrome was made based on the clinical picture. Emergency left thigh fasciotomy and assessment of fascia was performed, however, the muscle appeared pink and viable. Biopsy was performed and culture was taken. The wound was closed.

During the postoperative days, increased swelling and edema with bleeding was noticed from the surgical wound, accompanied by a significant drop in hematocrit and hemoglobin $20.6 \%$ and $6.7 \mathrm{~g} / \mathrm{dL}$, respectively requiring blood transfusion. The patient was taken back to the operating room for wound exploration where multiple hematomas within viable muscle were found. The patient required a third wound exploration due to recurrent blood clots and infarcted muscle was debrided until viable muscle with good perfusion was noted. The lateral fascia appeared to be necrotic and was debrided as well.

We retrieved his medical records from the previous hospital where he had multiple readmissions. Results of the MRI scan and muscle biopsy from the right thigh performed six month prior to this admission were reviewed. Axial $\mathrm{T} 1$ and fat suppressed proton-density images MRI scan revealed collections within the right vastus lateralis muscle and within the abductor muscle group, with signal compatible with the presence of blood and hemosiderin, indicative of hemorrhage (Figures 3 and 4). Debridement and biopsy were performed on the right thigh due to these "spontaneous hematomas". Histological examination revealed a focus of complete necrosis of muscle fibers, granulation tissue and phagocytosis. As per the pathologist interpretation these findings were consistent with "infarct of the thigh, a classical pathological process that causes a thigh mass, typically found in DM.

The biopsy report done at our institution was also consistent with muscle necrosis (Figure 5). We finally came to the conclusion that this was a case of diabetic muscle infarction (DMI).

Our patient had a long recovery period due to the recurrent spontaneous hematomas which required multiple visits to the operating room for muscle debridement, placement of wound-vac and evaluation by plastic surgery for possible skin graft.

\section{DISCUSSION}

Diabetic muscle infarction or myonecrosis is a rare complication of long standing DM, most commonly affecting type $1 \mathrm{DM}$, but can be seen in type $2 \mathrm{DM}$ as well. Since 1965, when Angervall et al. [2] first described

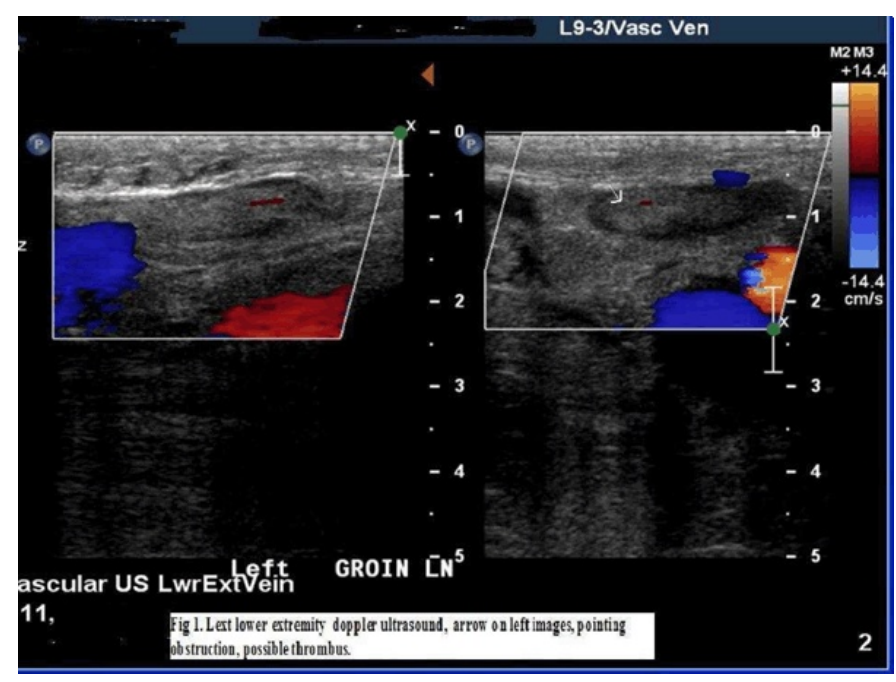

Figure 1: Left lower extremity Doppler ultrasound, arrow on left images, pointing obstruction, possible thrombus. 


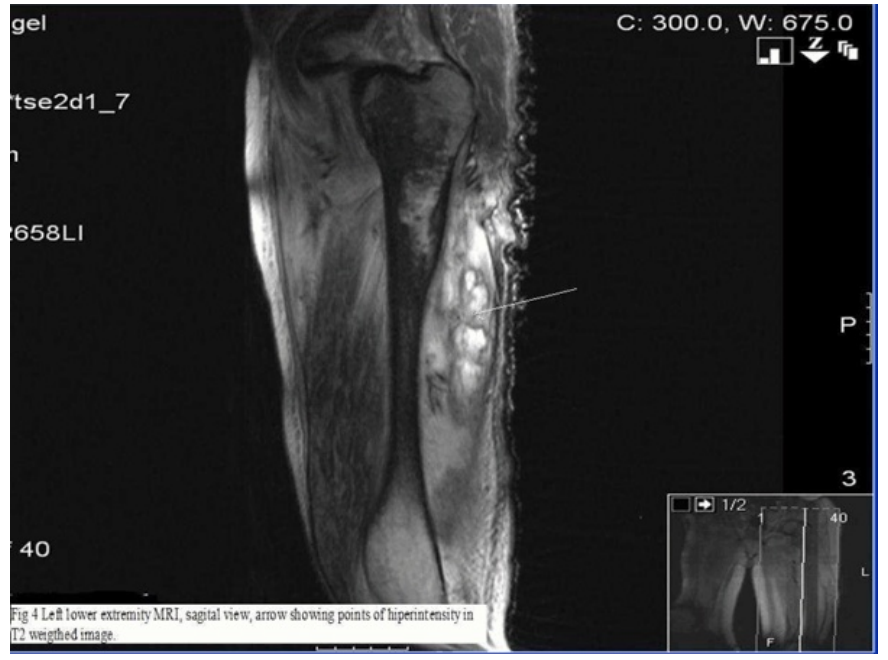

Figure 2: Left lower extremity venogram, prior to thrombectomy, left iliac vein.

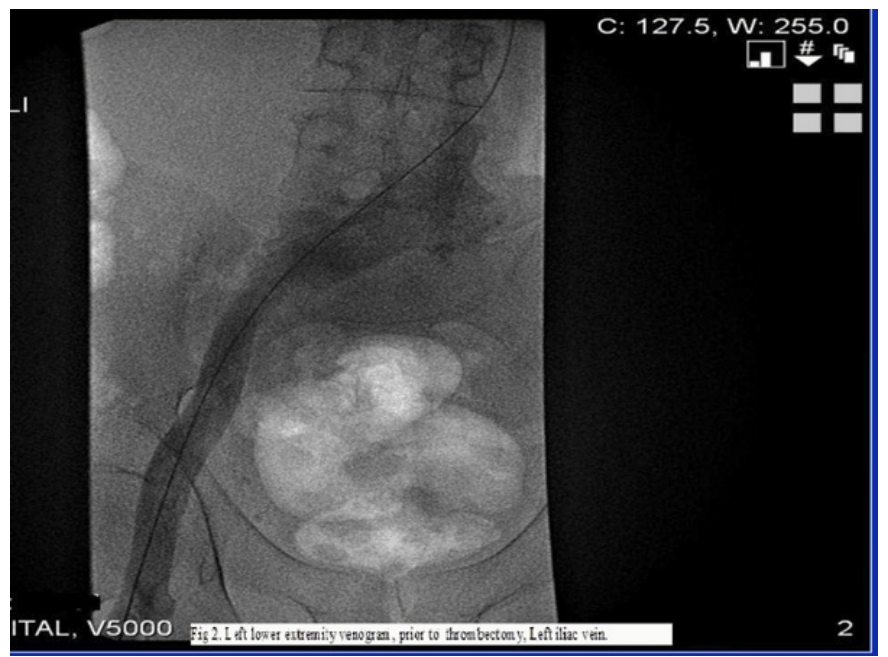

Figure 3: Left lower extremity magnetic resonance imaging, without contrast, axial view; arrow pointing to area of necrosis.

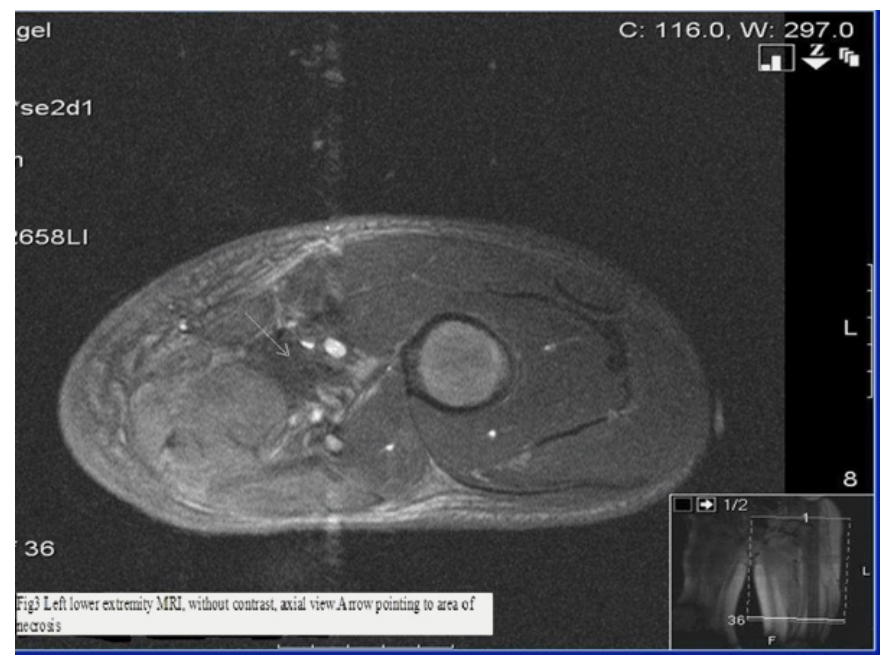

Figure 4: Left lower extremity magnetic resonance imaging, Sagittal view, arrow showing points of hiperintensity in T2 weighted image.

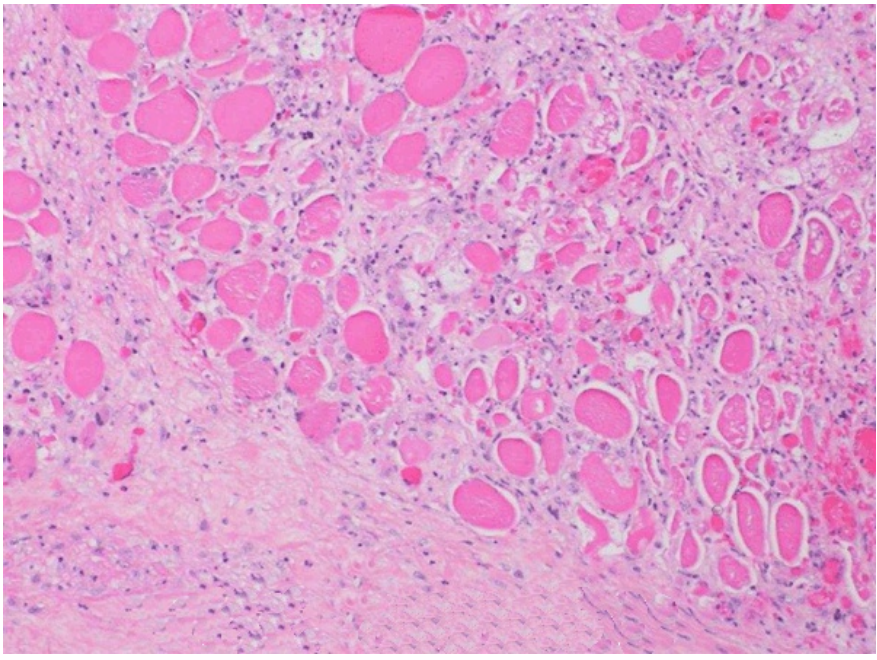

Figure 5: Muscle biopsy showing macrophages infiltration (H\&E stain, x100).

the pathology as tumoriform focal muscular degeneration, several case reports and series have been reported [3-5]. DMI has a tendency to affect the muscles of the lower extremities, especially the quadriceps, hip adductors and gastrocnemius. Our patient presented with left thigh pain and swelling, with previous similar presentations in the contralateral side.

Similar to other cases described in literature, our patient had been a poorly controlled diabetic with microvascular complications and ESRD on hemodialysis $[1,5,6]$. In a series by Lentine et al., it was observed that DMI is more common in hemodialysis patients versus peritoneal dialysis patients, which may reflect a triggering effect of hemodialysis on muscle infarction [7].

As for the pathogenesis, several hypotheses have been proposed. The infarction could be due to atherosclerosis and diabetic microangiopathy. Conversion of the normal rich collateral circulation of muscle to an end-vessel circulatory pattern renders it particularly vulnerable to injury [8]. It is possible that the reason practitioners often obviate this manifestation of diabetes may be due to poor understanding of its pathogenesis and significance. In a study by TrujilloSantos et al., many mechanisms are proposed, including an alteration of the coagulation-fibrinolysis system and vascular disease [5]. There is evidence of abnormal coagulation and fibrinolytic pathways in DMI, and the presence of antiphospholipid antibodies has been described in two cases. Unfortunately, no prospective studies regarding the use of anticoagulation have been conducted [9] In our case, the patient had an elevation of factor VIII, which may contribute to the hypercoagulable state theory [10].

The presenting symptoms were similar to what has been described in other reports: acute onset of lower extremity painful swelling, no prior trauma, no fever. However, in contrast, our case had multiple admissions to our hospital and other facilities with similar presentation, and was managed as a case of cellulites. 
A year prior to this admission an MRI scan was done showing soft tissue fluid in relation to the extensor muscles of the proximal thigh particularly around the rectus femoris muscle in the right tensor fascia lata. No conclusive diagnosis was made at that time. On this admission, patient was thought to have DVT at first, which is one of the differential diagnoses of this pathology along with pyomyositis, myositis ossificans, traumatic muscle rupture, muscle hemorrhage, fasciitis, osteomyelitis, abscess and soft tissue neoplasm [5]. Venogram was negative. The case was also compicated by the suspicion of atraumatic compartment syndrome.

To diagnose DMI the clinician must keep it high in the differential diagnosis, especially in poorly controlled DM patients with late complications such as nephropathy who present with lower extremity pain. Laboratory tests are helpful in ruling out other possible differential diagnosis. It has been reported in many cases that acute phase reactants tend to be elevated and creatine kinase (CPK) could be normal to mildly elevated. In our case CPK was mildly elevated.

The MRI scan is diagnostic test of choice since it can detect the characteristic changes with high sensitivity. A hyper-intense T2-weighted muscle signal, a reflection of increased tissue water, is the most common finding [7]. Muscle biopsy may clarify the diagnosis when clinical findings support several etiologies. Histopathological changes in DMI vary with timing of biopsy acquisition from acute coagulative necrosis with inflammatory infiltrates to myofiber atrophy and fibrosis. Patient had a prior biopsy with a positive result.

In reviews, the majority of cases resolved spontaneously only requiring symptomatic treatment which includes non-steroidal anti-inflammatory drugs, rest, adequate glycemic control and physical therapy. This is established as the standard of care, although pathology can definitely re-occur as seen in our patient. Surgery and biopsy can delay recovery, as occurred with our case, leading to increase morbidity and decreased quality of life [10].

\section{CONCLUSION}

Although rare, DMI is a serious and potentially disabling complication of uncontrolled diabetes mellitus which continues to be under diagnosed. Hospitalists and primary care physicians should be educated extensively on this entity, keeping it among the differential diagnosis of the diabetic patient with non-traumatic leg pain and swelling. Recognizing diabetic muscle infarction more efficiently could save the patient from undergoing unnecessary, diagnostic procedures, including medical or surgical interventions that could in turn result in more harm than good to the patient. Being able to correctly diagnose these patients and educating them on the nature of their condition may also aid in reducing medical costs. Our recommendation for diabetic patients with microvascular disease, presenting with leg swelling and pain, is that once deep venous thromboembolism is ruled out, magnetic resonance imaging should be done in order to diagnose this diabetic muscle infaction.

\section{ACKNOWLEDGEMENTS}

Meno Lueders, MD, Department of Surgery

Kimberly Andrews-Reynolds, MD, Research Assistant

$$
* * * * * * * * *
$$

\section{Author Contributions}

Sarah Olivier - Conception and design, Acquisition of data, Analysis and interpretation of data, Drafting the article, Critical revision of the article, Final approval of the version to be published

Raquel Michel - Conception and design, Acquisition of data, Analysis and interpretation of data, Drafting the article, Critical revision of the article, Final approval of the version to be published

Aracelis Lu - Acquisition of data, Drafting the article, Final approval of the version to be published

Vihren Dimitrov - Conception and design, Analysis and interpretation of data, Critical revision of the article, Final approval of the version to be published

\section{Guarantor}

The corresponding author is the guarantor of submission.

\section{Conflict of Interest}

Authors declare no conflict of interest.

\section{Copyright}

(C) Sarah Olivier et al. 2013; This article is distributed under the terms of Creative Commons attribution 3.0 License which permits unrestricted use, distribution and reproduction in any means provided the original authors and original publisher are properly credited. (Please see www.ijcasereportsandimages.com/copyright-policy.php for more information.)

\section{REFERENCES}

1. Morcuende JA, Dobbs MB, Crawford H, Buckwalter JA. Diabetic muscle infarction. Iowa Orthop Journal 2000;20:65-74.

2. Angervall L, Stener B. Tumoriform focal muscular degeneration in two diabetic patients. Diabetologia 1965;1:39-42.

3. Maclsaac RJ, Jerums G, Scurrah L. Diabetic muscle infarction. Med J Aust 2002;177(6):323-4.

4. Bunch TJ, Birskovich LM, Eiken PW. Diabetic myonecrosis in a previously healthy woman and review of a 25-year Mayo Clinic experience. Endocr Pract 2002;8(5):343-6.

5. Trujillo-Santos AJ. Diabetic muscle infarction: An underdiagnosed complication of long-standing diabetes. Diabetes Care 2003 Jan;26(1):211-5. 
6. Reyes-Balaguer J. Solaz-Moreno E, Morata-Aldea C, Elorza-Montesinos P. Spontaneous diabetic myonecrosis. Diabetes Care 2005 Apr;28(4):980-1.

7. Lentine KL, Guest SS. Diabetic muscle infarction in end-stage renal disease. Nephrol Dial Transplant 2004 Mar;19(3):664-9.

8. Anderson WR, Richards AM. Evaluation of lower extremity muscle biopsies in the diagnosis of atheroembolism. Arch Pathol 1968;86(5):535-41.

9. Palmer GW, Greco TP. Diabetic thigh muscle infarction in association with antiphospholipid antibodies. Semin Arthritis Rheum 2001;30(4):272-80.

10. Bjornskov EK, Carry MR, Katz FH, Leflowitz J, Ringel SP. Diabetic muscle infarction: a new perspective on pathogenesis an management. Neuromuscul Disord 1995 Jan;5(1):39-45.

Access full text article on other devices

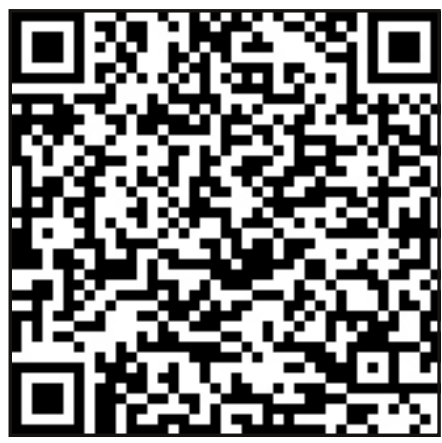

Access PDF of article on other devices

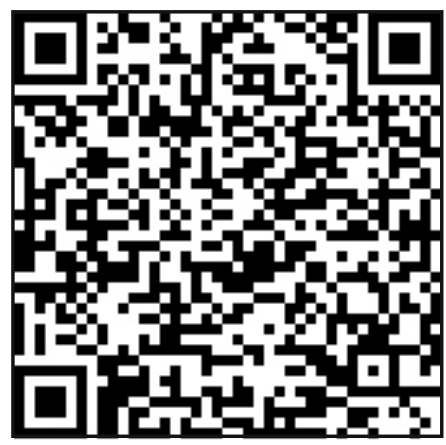

